\title{
FishDB: an integrated functional genomics database for fishes
}

\author{
Liandong Yang ${ }^{1}$, Zetan $\mathrm{Xu}^{2}$, Honghui Zeng ${ }^{1}$, Ning Sun ${ }^{1,3}$, Baosheng $\mathrm{Wu}^{3,4}$, Cheng Wang ${ }^{1,3}$, Jing Bo ${ }^{3,4}$, Lin $\mathrm{Li}^{1,3}$, \\ Yang Dong ${ }^{2}$ and Shunping $\mathrm{He}^{1,4,5^{*}}$
}

\begin{abstract}
Background: Hundreds of genomes and transcriptomes of fish species have been sequenced in recent years. However, fish scholarship currently lacks a comprehensive, integrated, and up-to-date collection of fish genomic data.

Results: Here we present FishDB, the first database for fish multi-level omics data, available online at http://fishdb. ihb.ac.cn. The database contains 233 fish genomes, 201 fish transcriptomes, 5841 fish mitochondrial genomes, 88 fish gene sets, 16,239 miRNAs of 65 fishes, 1,330,692 piRNAs and 4852 IncRNAs of Danio rerio, 59,040 Mb untranslated regions (UTR) of 230 fishes, and $31,918 \mathrm{Mb}$ coding sequences (CDS) of 230 fishes. Among these, we newly generated a total of 11 fish genomes and 53 fish transcriptomes.
\end{abstract}

Conclusions: This release contains over $410,721.67 \mathrm{Mb}$ sequences and provides search functionality, a BLAST server, JBrowse, and PrimerServer modules.

Keywords: Fish, Genome, Transcriptome, Evolution, Adaptation

\section{Background}

Fish are the largest group of vertebrates, covering over one-half of the world's living vertebrates [1]. Considering the vast diversity of species and morphology, fish have received intense attention from scholars and the public, as they are important to both scientific research and aquaculture. The availability of fish genomes and transcriptomes will provide valuable resources for ichthyological research. However, fish scholarship currently lacks a comprehensive, integrated, up-to-date collection of fish omics data.

Currently, at least 222 fish genomes have been sequenced and deposited in public databases, including the NCBI genome database [2], Ensembl [3], UCSC [4],

\footnotetext{
* Correspondence: clad@ihb.ac.cn

'State Key Laboratory of Freshwater Ecology and Biotechnology, Institute of Hydrobiology, Chinese Academy of Sciences, Wuhan 430072, China

${ }^{4}$ Institute of Deep-sea Science and Engineering, Chinese Academy of Sciences, Sanya 572000, China

Full list of author information is available at the end of the article
}

SalmoBase [5], GCGD [6], and cBARBEL [7]. Because the cost of whole-genome sequencing has decreased, many whole-genome sequencing projects on fish have been performed in recent years, usually by small research groups with technical support from private companies. Although the genomes are often required to be submitted to the NCBI genome database before publication, the database is not scheduled to be updated frequently in the future. More importantly, many more genomes have already been sequenced, but the necessary studies take time to publish, leaving many genomes unavailable to the research community. In this way, ichthyological research has been severely hampered for lack of a comprehensive, integrated, and up-to-date collection of fish omics database.

Here, we generated FishDB (http://fishdb.ihb.ac.cn), which is intended to meet the needs of the fish scholarship community. It is especially suitable for studies on taxonomy, phylogeny, evolution, development, and agriculture. As far as we know, FishDB gathers almost all of

(c) The Author(s). 2020 Open Access This article is licensed under a Creative Commons Attribution 4.0 International License, which permits use, sharing, adaptation, distribution and reproduction in any medium or format, as long as you give appropriate credit to the original author(s) and the source, provide a link to the Creative Commons licence, and indicate if changes were made. The images or other third party material in this article are included in the article's Creative Commons licence, unless indicated otherwise in a credit line to the material. If material is not included in the article's Creative Commons licence and your intended use is not permitted by statutory regulation or exceeds the permitted use, you will need to obtain permission directly from the copyright holder. To view a copy of this licence, visit http://creativecommons.org/licenses/by/4.0/ The Creative Commons Public Domain Dedication waiver (http://creativecommons.org/publicdomain/zero/1.0/) applies to the data made available in this article, unless otherwise stated in a credit line to the data. 
Table 1 Summary of the data content of FishDB

\begin{tabular}{lll}
\hline Category & Species & Total Sequences $\mathbf{( M b )}$ \\
\hline Genome & 233 & $258,892.84$ \\
Transcriptome & 201 & $55,586.52$ \\
Mitogenomes & 5841 & 137.62 \\
EST & 136 & 3889.03 \\
Ortholog & 48 & 1218.18 \\
miRNA & 65 & 0.99 \\
piRNA & 1 & 33.81 \\
LnCRNA & 1 & 3.89 \\
UTR & 230 & $59,040.01$ \\
CDS & 230 & $31,918.78$ \\
\hline
\end{tabular}

its fish genomes and most of its fish transcriptomes from public databases. We also included a total of 11 fish genomes and 53 fish transcriptomes collected by our group, which have not been accessible previously. FishDB provides not only widely used web-services such as a search tool, BLAST, JBrowse, and PrimerServer, but also a platform for comparative genomics analysis on orthologs.

\section{Construction and content}

\section{Data sources}

FishDB integrates fish gene data from as many as dozens of databases (Table 1). Here, we generated a total of 11 fish genomes and 53 fish transcriptomes for the first time.

Most of the fish genomes were obtained from the genome database in NCBI [2], Ensembl [3], UCSC [4], EFish, SalmoBase [5], GCGD [6], and cBARBEL [7] (Supplementary Data S1). We also assembled 11 new genomes of comparable quality to those of the other fishes (Supplementary Data S2). All individual fish were euthanized, which was approved by the Institutional Animal Care and Use Committee of Institute of Hydrobiology, Chinese Academy of Sciences (Approval ID: Y21304501). Fish were purchased from a commercial aquarium (Wuhan Shengdajiahe Aquarium). One individual of each fish species was collected. Adult fishes were euthanized individually by immersion in water baths in a 5-L holding tank with aerated water containing $500 \mathrm{mg} / \mathrm{L}$ of MS-222 (Sigma). When the fish died, their muscle tissue was collected for sequencing. This generated a total of 233 fish genomes (Table 2). Among them, we obtained annotation files of genes for a total of 88 fish genomes (Supplementary Data S3) (Fig. 2a and b).

Assembled fish transcriptomes were downloaded from the NCBI TSA (Transcriptome Shotgun Assemblies) database (Supplementary Data S4). We generated a total of 53 new transcriptomes sampled from tissues including muscle, brain, liver, kidney, and heart, which were then assembled using Trinity [8] with default parameters (Supplementary Data S5). We further collected a total of 49,406 raw RNA-seq from NCBI SRA (Sequence Read Archive) database (Supplementary Data S6).

Fish mitochondrial genomes were collected from MitoFish (Mitochondrial Genome Database of Fish) [9, 10]. A total of 2726 complete mtDNA sequences from 2726 fish species were obtained. We further downloaded a total of 8094 complete mtDNA sequences from 3121 fish species (Supplementary Data S7).

Expressed sequence tags (ESTs) of 136 fish were obtained from the EST database in NCBI [2].

Fish orthologs were downloaded from Ensembl (release 96; May 2019) using BioMart [11] and a total of 19,310 orthologs between zebrafish and at least one other fish species were downloaded (Supplementary Data S8) (Fig. 2c).

Fish miRNA sequences were collected from the miRBase [12], Ensembl (release 96; May 2019), and also obtained from the supplemental materials of published references when the miRNA sequences were not deposited into miRBase. In total, the miRNAs from 65 fish were stored in FishDB (Supplementary Data S9).

For piRNA and long noncoding RNA (lncRNA), a total of 1,330,692 piRNAs and 4852 lncRNAs of Danio rerio

Table 2 The distribution of fish genome resource

\begin{tabular}{llll}
\hline Database & Species & Genome with Gene Sets & URL \\
\hline NCBI (Genome) & 294 & 74 & https://www.ncbi.nlm.nih.gov/genome/ \\
Ensembl & 48 & 48 & https://asia.ensembl.org/index.html \\
UCSC & 10 & 10 & https://genome.ucsc.edu/ \\
EFish & 3 & 2 & https://efishgenomics.integrativebiology.msu.edu/ \\
SalmoBase & 2 & 2 & https://salmobase.org/ \\
GCGD & 1 & 1 & http://bioinfo.hbb.ac.cn/gcgd/php/index.php \\
CBARBEL & 1 & 1 & http://catfishgenome.org \\
New generated & 11 & 11 & - \\
FishDB & 303 & 91 & http://fishdb.ihb.ac.cn \\
\hline
\end{tabular}




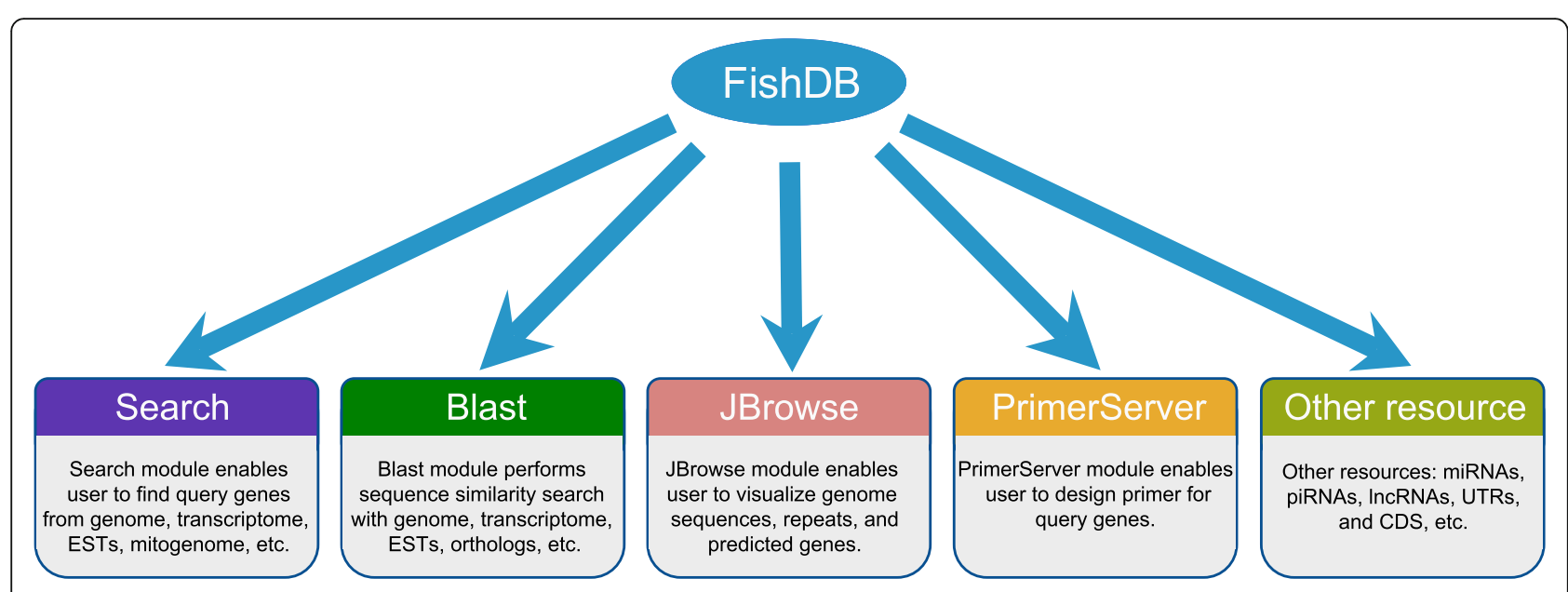

Fig. 1 The structure of FishDB, which provides Search, Blast, JBrowse, PrimerServer, Ortholog, and fish gene information

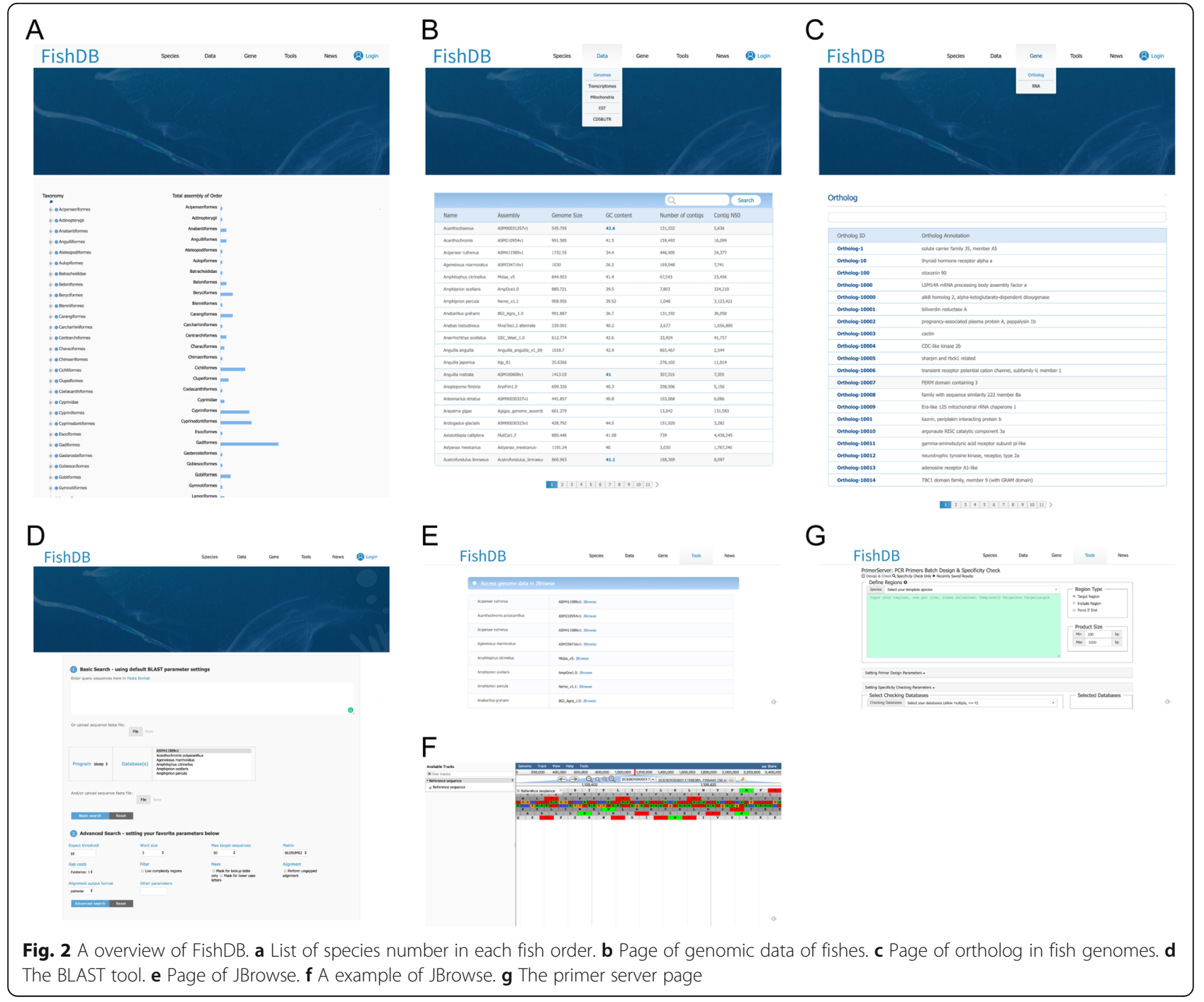


were downloaded from piRBase [13] and NONCODE [14], respectively.

Coding sequences (CDS) and untranslated regions (UTR).

We also obtained UTR sequences of five fish from the UTRBase [15]: Danio rerio, Oncorhynchus mykiss, Oryzias latipes, Salmo salar, and Takifugu rubripes. We also predicted CDS and UTR using TransDecoder from transcriptome sequences, producing CDS and UTR sequences for a total of 230 fish. In addition, we obtained CDS and UTR sequences from 48 fish genomes predicted by Ensembl. Collectively, CDS and UTR sequences from a total of 230 fish species were collected in FishDB (Supplementary Data S10).

\section{Utility and discussion}

\section{Structure of FishDB}

FishDB offers web services including a search tool, BLAST, JBrowse, and PrimerServer. The gene information for noncoding RNA (ncRNA), microRNA (miRNA), UTRs, and CDSs was collected and stored in the FishDB database (Fig. 1).

\section{Search}

The search module enables the user to collect interesting information, such as sequences, from genomes, transcriptomes, genes, ESTs, mitogenomes, and orthologos using either a gene name or geneID. In addition to sequence information, users could also find relevant information from the gene. The search results provide users with links to NCBI records.

\section{Blast}

The blast module performs sequence similarity search employing a web-based BLAST server [16]. Users can use nucleotide BLAST (BLASTN and TBLASTN) searches against the 233 fish genomes, 201 fish transcriptomes, 136 fish ESTs, and 88 fish OGSs, and they can use amino acid BLAST (BLASTX and TBLASTX) searches against the 88 fish protein sequences (Fig. 2d).

\section{JBrowse}

The JBrowse module enables users to visualize the 88 fish genomes [17], which is a related browser to the conventional CGI-based genome browser (GBrowse). This genome browser enables users to find and explore the 88 fish genome sequences and annotation information easily. Three main tracks, including CDS, mRNA, and exon, are integrated for all fish genomes. Users can find various tracks and search genomic features inside in the reference genome, including transposable elements,gene models, and repeats (Fig. 2e and f).

\section{PrimerServer}

The PrimerServer module helps users design primers that are particular to polymerase chain reaction experiments (PCR). We used Primer3 [18] to produce candidate primer pairs for the sequences of given template. We also integrated Primer Blaster, a specific tool, to test the specificity of each primer pair. The designed primer sequences can be downloaded as fasta format. (Fig. 2g).

\section{Conclusions}

We have built the Fish Genome Database (FishDB), which provides a central portal for genomics, transcriptomics, genetics, and evolutionary biology of fish. FishDB stores various sequences, including genomes, transcriptomes, mitochondrial genomes, ESTs, orthologs, noncoding RNAs, UTRs, and CDSs of fish species. The database also provides query, visualization, and primer design tools including BLAST, JBrowse, and PrimerServer. FishDB will be continuously updated when new genome, transcriptome, and genetic datasets of fish become available, and more enhanced functionality will be possible in the future to generate a more valuable resource for promoting comparative genomics, transcriptomes, and evolutionary biology studies.

\section{Supplementary Information}

The online version contains supplementary material available at https://doi. org/10.1186/s12864-020-07159-9.

Additional file 1 : Supplementary Data S1. The fish genomes downloaded from public databases in FishDB.

Additional file 2 : Supplementary Data S2. The fish genomes newly generated from our lab in FishDB.

Additional file $\mathbf{3}$ : Supplementary Data S3. The fish gene sets in FishDB.

Additional file 4 : Supplementary Data S4. The fish transcriptomes downloaded from Transcriptome Shotgun Assembly (TSA) in FishDB.

Additional file $\mathbf{5}$ : Supplementary Data S5. The fish transcriptomes newly generated from our lab in FishDB.

Additional file 6 : Supplementary Data S6. The RNA-seq data sets of fish transcriptomes downloaded from Sequence Read Archive (SRA) in FishDB.

Additional file 7 : Supplementary Data S7. The fish mitochondrial genomes obtained from MitoFish and NCBI in FishDB.

Additional file 8 : Supplementary Data S8. Orthologs between zebrafish and at least one other fish species from Ensembl.

Additional file 9 : Supplementary Data S9. The fish miRNAs in FishDB.

Additional file 10 : Supplementary Data S10. The UTRs and CDSs of fishes in FishDB.

\section{Abbreviations}

IncRNAs: Long non-coding RNAs; Mb: Millions of base pairs;

UTR: Untranslated region; CDS: Coding sequences; TSA: Transcriptome shotgun assemblies; SRA: Sequence read archive; ESTs: Expressed sequence tags 


\section{Acknowledgements}

We thank Nowbio Biotechnology Company (Kunming, China) for their support in database construction.

\section{Authors' contributions}

S. H., Y.D. and L.Y. conceived this study. Z. X. and H. Z. constructed the database. L.Y., N.S., B.W., C.W., J.B., and L.L. collected and analyzed the datasets. All authors have read and approved the manuscript.

\section{Funding}

This research was supported by the Strategic Priority Research Program of Chinese Academy of Sciences (XDB31000000) and the National Natural Science Foundation of China (31972866). This research was supported by the Wuhan Branch, Supercomputing Center, Chinese Academy of Sciences, China.

\section{Availability of data and materials}

FishDB can be accessed at http://fishdb.ihb.ac.cn. All data used in this study are available from Supplementary Data S1, S3, S4, S6, S7, and S8.

\section{Ethics approval and consent to participate}

All this study was submitted to and approved by the Institutional Animal Care and Use Committee of Institute of Hydrobiology, Chinese Academy of Sciences (Approval ID: Y21304501).

\section{Consent for publication}

This database contains no personal data.

\section{Competing interests}

The authors declare that they have no competing interests.

\section{Author details}

'State Key Laboratory of Freshwater Ecology and Biotechnology, Institute of Hydrobiology, Chinese Academy of Sciences, Wuhan 430072, China. ${ }^{2}$ State Key Laboratory for Conservation and Utilization of Bio-Resources in Yunnan, Yunnan Agricultural University, Kunming 650201, China. ${ }^{3}$ University of Chinese Academy of Sciences, Beijing 100049, China. ${ }^{4}$ Institute of Deep-sea Science and Engineering, Chinese Academy of Sciences, Sanya 572000, China. ${ }^{5}$ Center for Excellence in Animal Evolution and Genetics, Chinese Academy of Sciences, Kunming 650223, China.

Received: 18 November 2019 Accepted: 19 October 2020

Published online: 17 November 2020

\section{References}

1. Nelson JS, Grande TC, Wilson MVH. Fishes of the world. Hoboken: Wiley; 2016.

2. Sayers EW, Agarwala R, Bolton EE, et al. Database resources of the Nationa Center for biotechnology information. Nucleic Acids Res. 2019:47:D23-8.

3. Cunningham F, Achuthan P, Akanni W, et al. Ensembl 2019. Nucleic Acids Res. 2019:47:D745-51.

4. Haeussler M, Zweig AS, Tyner $C$, et al. The UCSC genome browser database: 2019 update. Nucleic Acids Res. 2019;47:D853-8.

5. Samy JKA, Mulugeta TD, Nome T, et al. SalmoBase: an integrated molecular data resource for salmonid species. BMC Genomics. 2017;18:482.

6. Chen Y, Shi M, Zhang W, et al. The grass carp genome database (GCGD): an online platform for genome features and annotations. Database. 2017;2017: bax051.

7. Lu J, Peatman E, Yang Q, et al. The catfish genome database CBARBEL: an informatic platform for genome biology of ictalurid catfish. Nucleic Acids Res. 2011;39:D815-21.

8. Grabherr MG, Haas BJ, Yassour M, et al. Full-length transcriptome assembly from RNA-Seq data without a reference genome. Nat Biotechnol. 2011;29: $644-52$

9. Iwasaki W, Fukunaga T, Isagozawa R, et al. MitoFish and MitoAnnotator: a mitochondrial genome database of fish with an accurate and automatic annotation pipeline. Mol Biol Evol. 2013:30:2531-40.

10. Sato Y, Miya M, Fukunaga T, et al. MitoFish and MiFish pipeline: a mitochondrial genome database of fish with an analysis pipeline for environmental DNA Metabarcoding. Mol Biol Evol. 2018;35:1553-5.
11. Smedley D, Haider S, Durinck S, et al. The BioMart community portal: an innovative alternative to large, centralized data repositories. Nucleic Acids Res. 2015;43:W589-98.

12. Kozomara A, Birgaoanu M, Griffiths-Jones S. miRBase: from microRNA sequences to function. Nucleic Acids Res. 2019;47:D155-62.

13. Wang J, Zhang P, Lu Y, et al. piRBase: a comprehensive database of piRNA sequences. Nucleic Acids Res. 2019;47:D175-80.

14. Zhao Y, Li H, Fang S, et al. NONCODE 2016: an informative and valuable data source of long non-coding RNAs. Nucleic Acids Res. 2016;44:D203-8.

15. Grillo G, Turi A, Licciulli F, et al. UTRdb and UTRsite (RELEASE 2010): a collection of sequences and regulatory motifs of the untranslated regions of eukaryotic mRNAs. Nucleic Acids Res. 2010;38:D75-80.

16. Mount, D.W. (2007) Using the Basic Local Alignment Search Tool (BLAST). CSH Protoc., pdb.top17.

17. Skinner ME, Uzilov AV, Stein LD, et al. JBrowse: a next-generation genome browser. Genome Res. 2009;19:1630-8.

18. Koressaar T, Remm M. Enhancements and modifications of primer design program Primer3. Bioinformatics. 2007;23:1289-91.

\section{Publisher's Note}

Springer Nature remains neutral with regard to jurisdictional claims in published maps and institutional affiliations.
Ready to submit your research? Choose BMC and benefit from:

- fast, convenient online submission

- thorough peer review by experienced researchers in your field

- rapid publication on acceptance

- support for research data, including large and complex data types

- gold Open Access which fosters wider collaboration and increased citations

- maximum visibility for your research: over $100 \mathrm{M}$ website views per year

At BMC, research is always in progress.

Learn more biomedcentral.com/submissions 